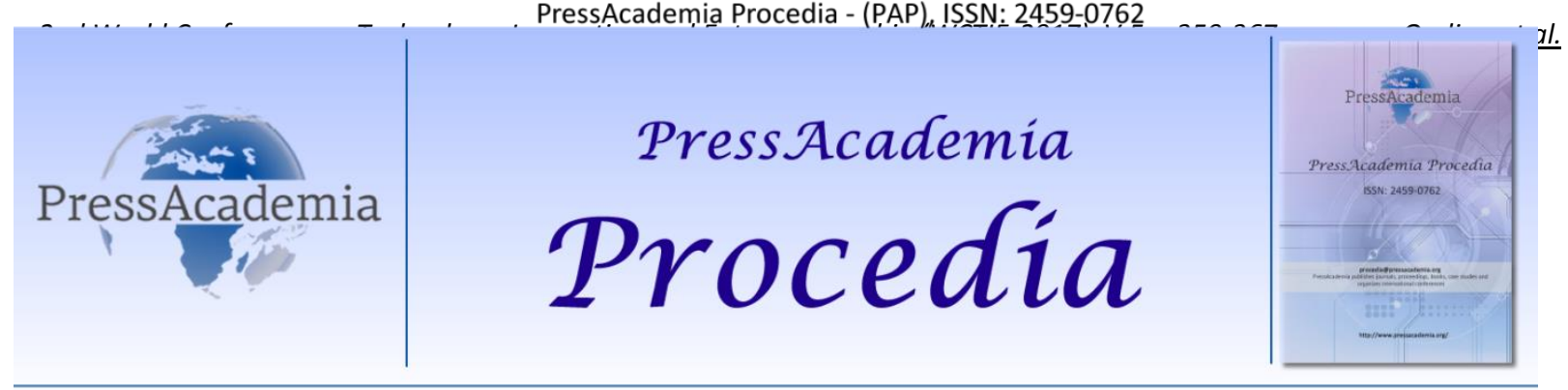

2nd World Conference on Technology, Innovation and Entrepreneurship

May 12- 14, 2017, Istanbul, Turkey. Edited by Sefer Şener

\title{
SIMULATION OF THE RADIO-INDUIT DAMAGES BY PHOTONS ON THE DNA MOLECULE IN ITS ENVIRONMENT
}

\section{DOI: 10.17261/Pressacademia.2017.611}

PAP-WCTIE-V.5-2017(49)-p.359-367

H.Oudira ${ }^{1}$; D. Lotfi ${ }^{1} ;$ A.Saifi $^{2}$

${ }^{1}$ Faculty of Technology, Department of Electronics University Mouhamed Boudiaf of M'Sila , Algeria. Oudira houcine@yahoo.fr

${ }^{2}$ Faculty of Technology, Department of Electronics University Mouhamed Boudiaf of M'Sila, Algeria. lotfi_djouane@yahoo.fr

${ }^{3}$ Faculty of Engineering Department of Electronics University of Constantine 1, Algeria. Saifi19@yahoo.fr

\section{ABSTRACT}

Monte Carlo codes and equation of diffusion were successively, used to simulate the physical, and physico-chemical, and chemical steps that to estimate the simple and double strand breaks produced on a linear and hydrated DNA model by an incident photons beam. The increase of the biological effectiveness, between 150 and $550 \mathrm{eV}$, is mainly due to the variation of photon cross section upon the Kionisation threshold in $\mathrm{O}, \mathrm{N}, \mathrm{C}$ atoms of DNA. In this study we present yields of main radiolysis species $\left(\mathrm{e}_{\mathrm{aq}}^{-}, \mathrm{H}, \mathrm{OH}\right)$ as function of time, then parameterised by the concentration of solute such formicate ( $\left.\mathrm{HCOO}^{-}\right)$. Moreover, we show that, with the same number of absorbed photons, the evolution of breaks as a function of energy reaches a maximum around $450 \mathrm{eV}$.

Keywords : DNA, Monte Carlo, diffusion, yields, solute, photons.

\section{INTRODUCTION}

The interaction of a mono-energy photon beam with matter induces point energy deposition. The latter then participate in the formation of a group of radicals which, after diffusion and reaction with the molecules of biological interest (cytosine, adenine, guanine, thymine), cause lesions and consequently genetic alterations in the Molecule of DNA [1]. We consider in this work:

- $\quad$ A break simple stand (C.S.B) is recorded when a direct ionization takes place on the grouping sweetens

- A break stand-in stand (CDB) is defined by a rupture simple sprig on every chain of the stand-in helix at the inside of a maximum distance of 10 pairs of cousin [2].

The direct effect of the photon incidents are analyzed by the methods of Monte Carlo step by step [3], while the indirect effect is treated by of the equation of diffusion to calculate the concentrations and the radiochemical yields of the different radiolysis species. Although it is less precise, it has the double advantage of reducing computation time and memory space [4].

\section{MODEL DESCRIPTION}

To simulate the biologic environment, we consider linear DNA fragment of B type constituted of 41 pairs of bases, whose properties are summarized in the table 1 [5].

Table 1: Helical Properties of the DNA

\begin{tabular}{||l|l||}
\hline Description of the fragment of DNA [5] & large size, END \\
\hline Sense of rotation of the helix & right \\
\hline Angle of rotation of a tour (in degree) & 36 \\
\hline Axis of the helix & The pairs of basis \\
\hline Big furrow & large \\
\hline Small furrow & narrow \\
\hline Pas $(\mathrm{nm})$ & 0.338 \\
\hline
\end{tabular}


The hydration envelope is adjusted with the help of modelling molecular Biosym software [6]. The whole is relaxed by the molecular minimization algorithm using the method of the biggest slope of the system potential energy, and inserted in the centre of a three-dimensional grid containing the water bulk; and the formate concentration varying between 0 and 2 moles by liter of water. The incidental photons are uniformly issued and perpendicularly to a plane surface, to a distance of $8 \mathrm{~nm}$ of the DNA center, privileging the direct effect of the irradiation thus in this range of energy (150-550 eV) as it can noted in figure 1. It is necessary to note that at the time of simulation of the ionization and excitation processes, it is taken account of the heterogeneity of the middle as well as the diffusion of the radiolysis spices created to the neighbourhood of the volume targets.

Figure1: Geometry of the Irradiation of the Fragment of DNA

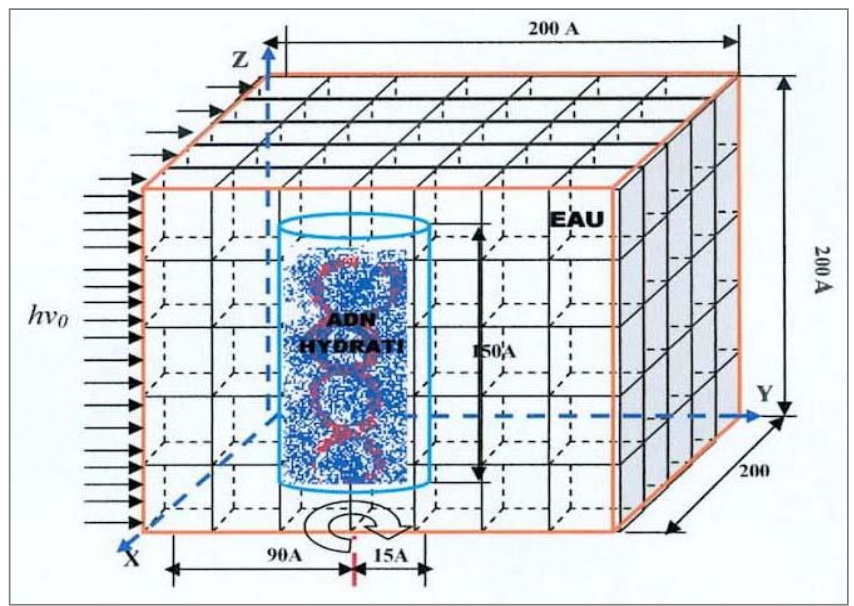

\section{MATIMATICAL MODELS}

\subsection{Stochastic Model of Monte Carlo}

The Monte Carlo method of the type step by step is adapted perfectly to the study of the physical and physic-chemical evaluation phases of the energy deposited in the target volume, to the extent that we consider the path $\lambda$ that an electron can traverse in a heterogeneous milieu and the probability of interaction with a given entity.

$$
\lambda=-\overline{\lambda(E)} \cdot \log R
$$

$\overline{\lambda(E)}$ is the range and is given by the relation:

$$
\overline{\lambda(E)}=\frac{1}{\sum_{i j} N_{i} \sigma_{i j}(E)}
$$

Where: $R$ is an arbitrary number equally distributed between 0 and 1 .

$N_{i}$ is the number of atoms or molecules of the type $i$ by unit of volume.

$\sigma_{i j}(E)$ is the effective section of interaction of the type $j$ on the atom or the molecule of the type $i$ for an incidental particle of energy $E$.

$$
P_{i}=\sigma_{i}(E) \cdot N_{i} \cdot \overline{\lambda(E)}
$$

$P_{i}$ is the probability for that an incidental particle interacts with an atom or a molecule of the $i$ component, knowing that there is interaction. 
If there are $k_{i}$ types of different interactions from the incidental particle with the $i$ component, the $j$ type of interaction having an effective section total $\sigma_{i j}(E)$ by atom or by molecule, the probability so that the interaction is of the type $j$, knowing that the interaction takes place with the $i$ component, is thus :

$$
P_{i j}=\frac{\sigma_{i j}(E)}{\sum_{l=1}^{k} \sigma_{i l}(E)}
$$

During simulation, the point of interaction being fixed, we will have an interaction with the $i$ component if $i$ verifies:

$$
\sum_{l=1}^{i-1} P_{l}<R_{1} \leq \sum_{l=1}^{i} P_{l}
$$

and the interaction will be of the type $j$ on the $i$ component if $j$ verifies:

$$
\sum_{l=1}^{j-1} P_{i l}<R_{2} \leq \sum_{l=1}^{j} P_{i l}
$$

$R_{1}$ and $R_{2}$ are arbitrary numbers equally distributed between 0 and 1 .

At the end of the physical stage $\left(10^{-15}\right.$ Second) the irradiated molecules are in an excited or ionized state [7] whose fate is drawn by probabilities indicated above.

at $10^{-12}$ second, the electronic trace produced, in its neighbor, the chemical species such as :

$$
e_{a q}^{-}, \mathrm{H}, \mathrm{OH}, \mathrm{H} 2, \mathrm{H} 2 \mathrm{O} 2 \text {, and } \mathrm{H}_{3} \mathrm{O}^{+} \text {. }
$$

With regard to, the cross sections of biological interest molecules [8], we adopted the correction introduced by Laverne and Pimblott [9].

$$
\sigma_{D N A}=\frac{A_{D N A}}{A_{\text {water }}} \cdot \sigma_{\text {water }}
$$

Where $A_{D N A}$ and $A_{\text {water }}$ are respectively the molar masses of the DNA and water.

Each atom of the DNA is differentiated from the other atoms by introduction of an empirical grandeur known as ray of Van Der Waals (RVDW). The total cross section $\sigma_{i}$ of an atom of VDW RVDW's ray is written:

$$
\sigma_{i}(E)=\frac{R_{V D W}^{i}}{\sum_{\text {Base }} R_{V D W}^{i}} \frac{A_{\text {Base }}}{A_{\text {water }}} \sigma_{\text {xater }}
$$

Thus, we exploited the rays of VDW presented by Burkert [10] for the simulation of the electrons transport in the biological environment.

\subsection{Diffusion Equation}

To reduce the calculating time machine, relating to the study of the chemical phase, we chose a deterministic approach based on the resolution of the diffusion equation [ 11 ].

$$
\frac{\partial C_{i}}{\partial t}=D_{i} \nabla^{2} C_{i}+S
$$


- $C i$ is the concentration of the species of the type $i\left(\right.$ mole $\left.\mathrm{dm}^{-3}\right)$

- $D_{i} \nabla^{2} C_{i}$ is the product of the Laplacian of $C_{i}$ by the constant of diffusion $D_{i}\left(\mathrm{~cm}^{2} \mathrm{~s}^{-1}\right)$ of the species of the type $i$;

- $S$ is the term source (mole $\mathrm{dm}^{-3} \mathrm{~s}^{-1}$ ), considered as the algebraic sum of terms representing, following the chemical reaction taken in consideration, the impoverishment or the production of the species of the type $i$ :

$$
S=\left(\sum_{w} k_{i w} C_{w}+\sum_{l} \sum_{j} k_{l j} C_{l} C_{j}\right)-\left(k_{i} C_{i}+\sum_{j} k_{i j} C_{i} C_{j}\right)
$$

$k_{i w} C_{w}, k_{l j} C_{l} C_{j}$ represent respectively the production of species $i$ following the dissociation of $w$, and the reaction of the species / with the species $j . k_{i} C_{i}$ and $k_{l j} C_{l} C_{j}$ represent respectively the impoverishment of species $i$ following its dissociation, and its reaction with the particles of the type $j ; k_{i w} k_{i}, k_{l j}$ and $k_{i j}$ indicate the constants of reaction's speed; $k_{i w}$ and $k_{i}$ are expressed in $\mathrm{s}^{-1}$, while $k_{l j}$ and $k_{i j}$ are in $\mathrm{dm} 3 \mathrm{~mole}^{-1} \mathrm{~s}^{-1}$.

\subsubsection{Discretizing the Diffusion Equation}

To solve the equation (9), it is necessary to discretize it, that is to say, to replace it by simple algebraic equations expressing the same physical information that can be solved numerically. For this, converts the equation (9) in an integral equation by applying the weighted residuals method:

$$
\iiint \int \frac{\partial C_{i}}{\partial t} W(X, Y, Z, t) d X d Y d Z d t=\iiint \int\left(D_{i} \nabla^{2} C_{i}+F\right) W(X, Y, Z, t) d X d Y d Z d t
$$

The choice of the test function $W(X, Y, Z, t)$, and integration of equation (11) cause algebraic equations such that the concentration $C_{i}$ in a point $\mathrm{P}\left(C_{i p}\right.$ noted $)$, the center of a elementary volume within the computational domain, is connected to those of the points $E, W, R, Q, S$, and $N$ center of neighboring elementary volumes considered (Figure 2).

Figure 2: Immediate Neighboring of an Elementary Volume Inside the Three-Dimensional Grid

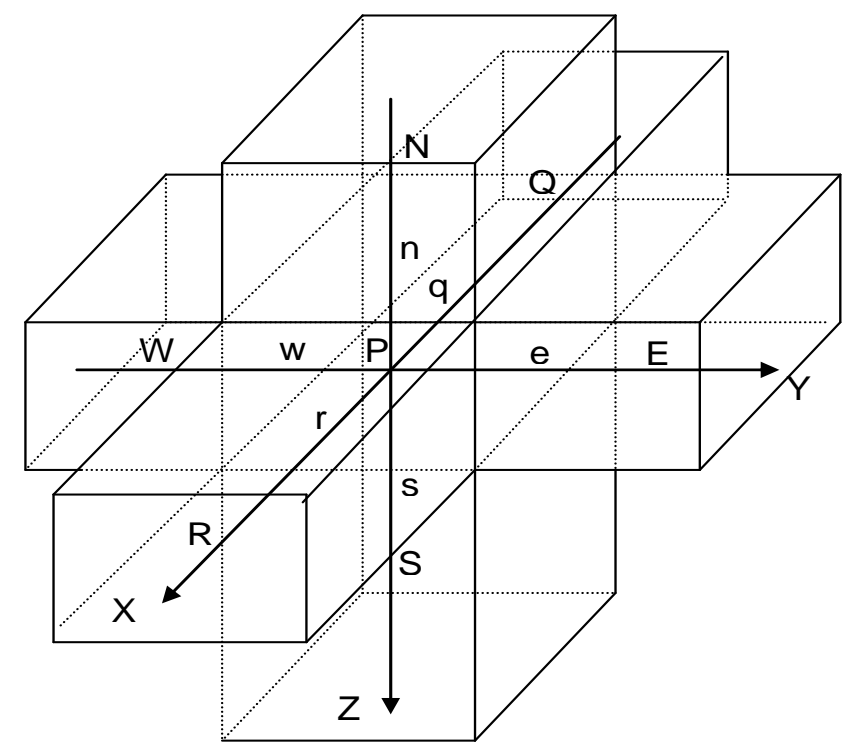

To access this formulation we have used the continuous finite element method [12], [13] and the method of centered differences [10]. Thus, the explicit method can replace the equation (11) by the following algebraic equation:

$$
A_{P} C_{i P}=A_{E} C_{i E}+A_{W} C_{i W}+A_{R} C_{i R}+A_{Q} C_{i Q}+A_{S} C_{i S}+A_{N} C_{i N}+K
$$




\subsubsection{Calculation of Equation (12) Coefficients:}

For a point $\mathrm{P}$ situated inside the three-dimensional grid, integrating (11) on the following ranges:

$[t ; t+\Delta t],\left[X_{P}-\frac{\Delta X}{2} ; X_{P}+\frac{\Delta X}{2}\right],\left[Y_{P}-\frac{\Delta Y}{2} ; Y_{P}+\frac{\Delta Y}{2}\right],\left[Z_{P}-\frac{\Delta Z}{2} ; Z_{P}+\frac{\Delta Z}{2}\right]$

provides the coefficients $A_{P}, A_{E}, A_{W}, A_{R}, A_{Q}, A_{S}, A_{N}$ and $K$ relating to the equation (12) and whose values:

$$
\begin{aligned}
& \mathrm{A}_{\mathrm{E}}=\mathrm{D}_{\mathrm{i}} \frac{\Delta \mathrm{X} \Delta \mathrm{Z}}{\Delta \mathrm{Y}}=\mathrm{A}_{\mathrm{W}} \\
& \mathrm{A}_{\mathrm{R}}=\mathrm{D}_{\mathrm{i}} \frac{\Delta \mathrm{Y} \Delta \mathrm{Z}}{\Delta \mathrm{X}}=\mathrm{A}_{\mathrm{Q}} \\
& \mathrm{A}_{\mathrm{S}}=\mathrm{D}_{\mathrm{i}} \frac{\Delta \mathrm{X} \Delta \mathrm{Y}}{\Delta \mathrm{Z}}=\mathrm{A}_{\mathrm{N}} \\
& \mathrm{A}_{\mathrm{P}}=\frac{\Delta \mathrm{X} \Delta \mathrm{Y} \Delta \mathrm{Z}}{\Delta \mathrm{t}}+\mathrm{A}_{\mathrm{E}}+\mathrm{A}_{\mathrm{W}}+\mathrm{A}_{\mathrm{R}}+\mathrm{A}_{\mathrm{Q}}+\mathrm{A}_{\mathrm{S}}+\mathrm{A}_{\mathrm{N}} \\
& \mathrm{k}=\frac{\Delta \mathrm{X} \Delta \mathrm{Y} \Delta \mathrm{Z}}{\Delta \mathrm{t}} \mathrm{C}_{\mathrm{iP}}^{0}+\mathrm{F}_{\mathrm{P}}^{0} \Delta \mathrm{X} \Delta \mathrm{Y} \Delta \mathrm{Z}
\end{aligned}
$$

$\Delta \mathrm{X}, \Delta \mathrm{Y}, \Delta \mathrm{Z}$ are spaces step in the three directions of the three-dimensional grid.

$\Delta t$ is the time after which the changes in the concentration of a given radiolytic species are evaluated. The boundary conditions on the extreme points of the domain used to associate their coefficients whose value varies according to the position of the elementary considered volume [10]. Following integration of equation (11) on all the elementary volumes of space concentrations, we obtain another matrix system by block, whose resolution by the method of generalized Thomas matrix calculation (called TDMA) is immediate [14]. Also, we give a detailed attention to the calculation of the space step $\Delta x$ whose value is reported with the temporal step $\Delta t$ to ensure the stability of the solutions of the equation of diffusion, to respect the electronic balance of the milieu as much as that are possible. This relation uses the constant of diffusion D of the fastest particle $\left(H_{a q}^{+}\right)$.

$$
(\Delta x)^{2} \geq \sqrt{4 D} \cdot \Delta t
$$

\section{DATA OF THE PROBLEM TO BE TREATED}

\subsection{Photoelectric Effective Sections}

In the range of considered energy (150-550 eV), almost $99.5 \%$ of the interactions are photoelectric [8]; also, at the time of the calculation of the free course and the different probabilities of interaction, we used the efficient sections established by Cullen [15], whose representation in Figure3 is corrected for the mean atomic composition of each element in a nucleotide of the nucleus, DNA. 
Figure 3: Photoelectric Efficient Sections Expressed by Nucleotide

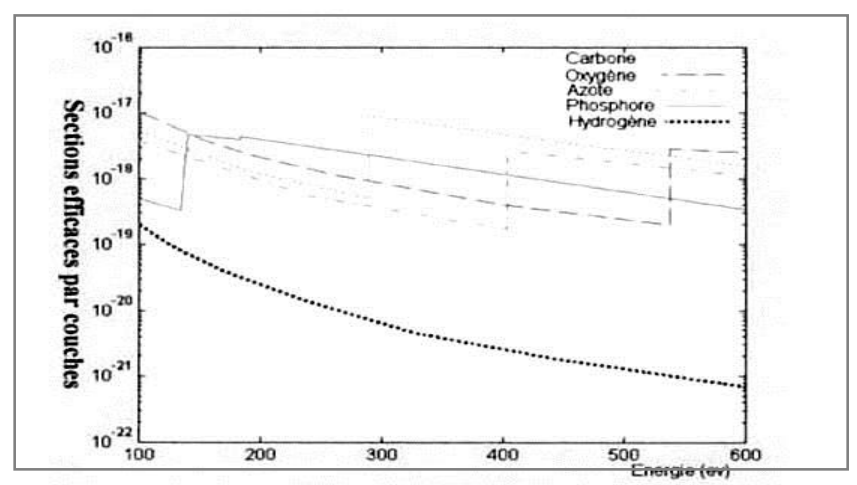

The middle atomic composition of our fragment of DNA est : Carbon 10, Oxygen 6, Nitrogen 4, Phosphor 1, hydrogen 11,

\section{RESULT AND DISCUSSION}

In this study, we were interested on the one hand, to the photoionizations produced on the carbon, nitrogen and oxygen atoms in view of the important proportions that they represent in the composition of the DNA and its envelope, On the other hand, to the outcome of the radicals created and their contributions to the formation of single and double-strand breaks as a function of energy.

\subsection{Direct Effect}

Between 0 and $600 \mathrm{eV}$, the free mean path is less than $1 \mathrm{~nm}$. This results lead to close interactions at the level of DNA and its hydration envelope. Indeed, as we can see in figure 4, between $250 \mathrm{eV}$ and $340 \mathrm{eV}$ the number of direct CSBs goes from one to two times of its initial value. This is due to the predominance of the number of carbon atoms per nucleotide, and to their cross-sections raised at this energy level. Moreover, between $350 \mathrm{eV}$ and $450 \mathrm{eV}$, the effect of the ionization threshold-K of the conjugated nitrogen at a relatively large proportion of atoms per nucleotide results in the creation of a maximum of CSB. Below the ionization threshold of carbon $(290 \mathrm{eV})$ and above the oxygen threshold $(540 \mathrm{eV})$, the photoionization is mainly done with liquid water, whose relatively small cross-sections confirm the number Restricted fractures at the level of the curve shown in figure 4.

\section{Figure 4: Simple Strand Breaks (CSB)d According to the Energy}

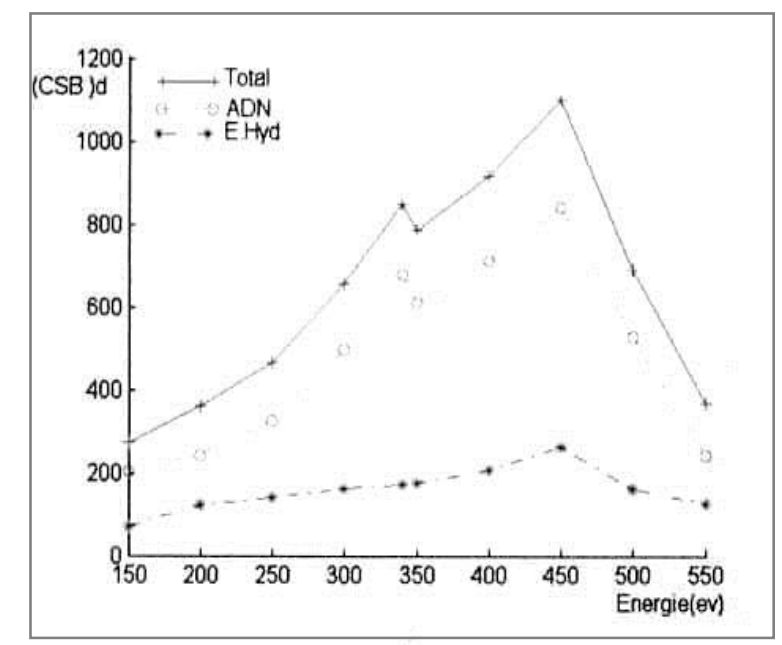

The photoelectrons and Auger electrons released in the surrounding water are the source of multiple ionizations leading to the formation of the $\mathrm{H}, \mathrm{OH}$ and $\mathrm{e}_{\mathrm{aq}}^{-}$radicals which indirectly react with the DNA subunits and its envelope of hydration to produce single and double strand breaks (Figure 5). 
The analysis of figure 5 shows that the single-strand breaks are greater in the hydration layer compared to those recorded at the DNA level, which has inspired us to undertake the study of the yields of the three main spices as a function of time (Figure 6).

It is deduced that the more the number of radicals is, the more the DNA subunits are attacked for a concentration $\Gamma$ and $C s$ low. The maximum protection of the DNA is reached for $\Gamma=20$ molecules of crystal water per nucleotide and $\mathrm{Cs}=0.7$ liter / mol of formate solute. Finally, we compare our results with those obtained by a purely stochastic method (Figure 7). An acceptable agreement between the breaks recorded by the two approaches is evident.

Figure 5: Indirect Simple Strand Breaks (CSB)i According to the Energy

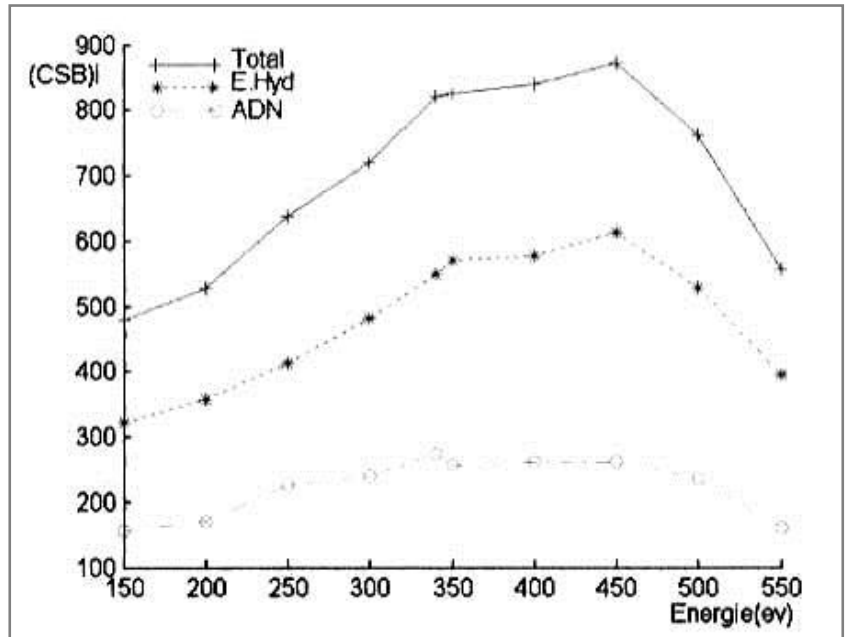

Figure 6: Radiochemical Yields of the Main Radiolytic Species as a Function of Time for Photons Energy of $290 \mathrm{eV}$

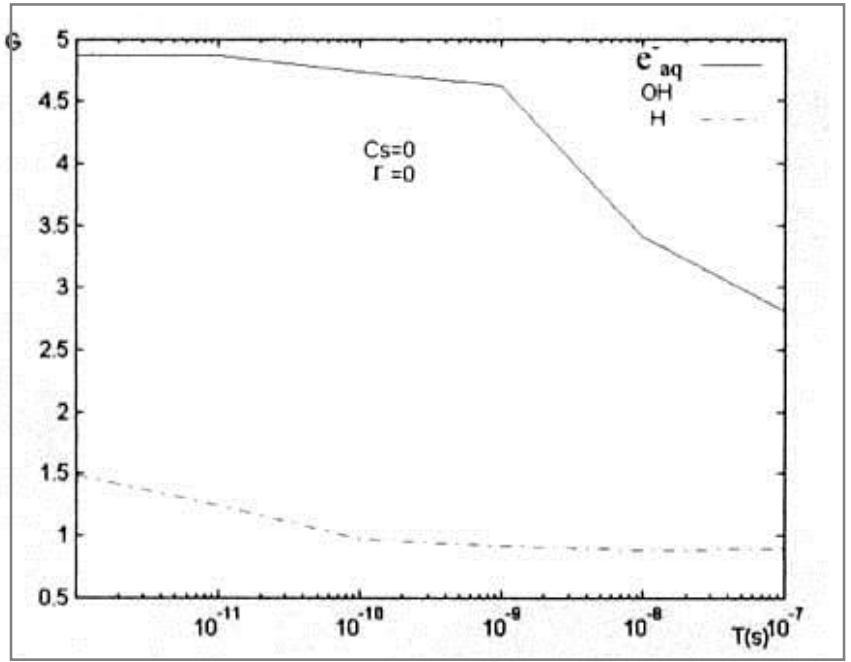


Figure 7: Normalized variations in relation to the peak value, of the damages recorded on a linear DNA model to $10^{-8} \mathrm{~s}$ for $\mathbf{2 0 0 0}$ absorbed photons. Comparison with the results of a merely stochastic model.

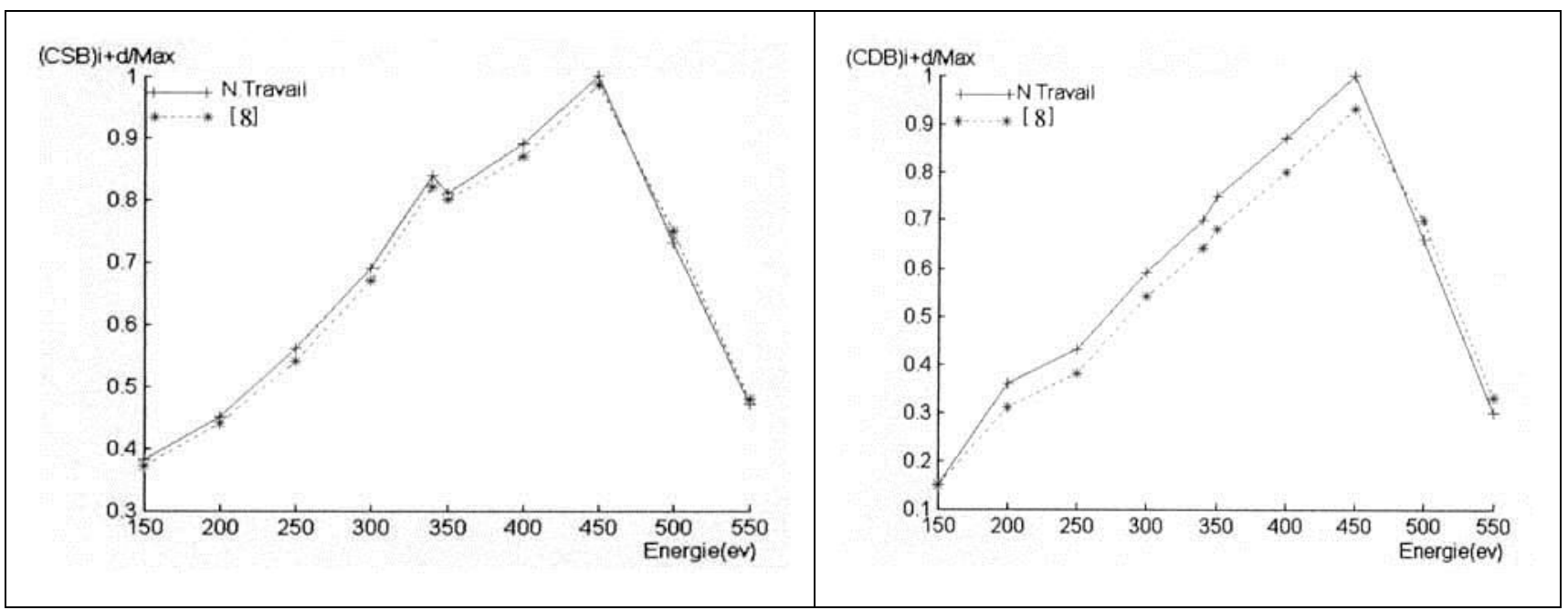

\section{CONCLUSION AND PERSPECTIVES}

Our conclusion to the evaluation of radio-induced damage at the level of the DNA molecule as a linear model is distinguished from those carried out numerically by Vrigneaud [8], in that it uses a deterministic method to study the Indirect effect of irradiation on the biological environment. This has the consequence in the elaborated software, the taking into account a maximum numbre of chemical reactions, a finest spatial step, while retaining the advantage of a lower calculation time. Moreover, the computer memory space helping, the extension of this method to the study of a more elaborate model such as the nucleosome is conceivable to approach a little more to the actual course of events.

\section{REFERENCES}

[1] - Saifi A.., Abdelmoumne M.E.K, Selmi S.,, "Survey of the products of the interaction of two distinct distributions of particles radiolytiques created at the time of the radiolyse of water by electrons ", Sc. \& Tech., Univ. of Constantine, N¹9 (2003), pp.36-44.

[2] - Vrigneaud J.M., "Development of a biophysical model for the radio damage assessment led in the chromosomal fiber", Thesis of university Doctorate, CPAT of Toulouse, (2000), p. 98.

[3] - Terrissol M., "Method of simulation of the transportation of electrons of included energies between 10eV and 30keV", Thesis of besciences Doctorate, CPAT of Toulouse, (1978).

[4] - Saifi A., "Resolution of the diffusion equation by a semi-implicit method. Application to the case of the water radiolyse by electrons", Radiop., Vol.30, n4, (1995), pp.557-573.

[5] - Demonchy M., "Modelling of the primary effect of the radiances on the DNA in his/her/its environment", Thesis of university Doctorate, CPAT of Toulouse, (1997), p.46.

[6] - Biosym / Molecular simulation, to use guides 95.0, San Diego, THAT.(1995).

[7]- Demonchy M., Ph.D, Modélisation de l'effet primaire des rayonnements sur l'ADN dans son environnement, T ( CPAT de Toulouse 1997).

[8] - Vrigneaud J.M., "Development of a biophysical model for the radio damage assessment led in the chromosomal fiber", Thesis of university Doctorate, CPAT of Toulouse, (2000), p. 98.

[9]- La Verne.J.M, Pumblott.S.M., Effect elastic collisions on energy deposition by electrons in water, J.Phys. chem..A, 1997, 101, 45044510.

[10]- Burkert.U, Allinger.N.L., Molecular Mechanics, ACS Monograh177, American chemical Society, Washington, D.C,1982. 
[11]- Oudira H., Djamai D., Saifi A., Application d'un modèle déterministe à l'étude de l'influence des molécules radioprotectrices sur les rendements des cassures simple et double brin de la molécule d'ADN, Radioprotection, 2008, 43, 389-408

[12]- CHATWITI .A. Ph.D, Etude de l'évolution temporelle des électrons dans un gaz faiblement ionisé, (Toulouse 1988).

[13]- ZIENKIEWICZ .O.C.,( 1988) " La méthode des éléments finis :McGraw-Hill,1971.

[14]- Boumahrat M., Gourdin A., (1983)."Méthodes numériques appliquée", Alger, OPU

[15] - Cullen D.E., "Tables and graphs of $\mu$ photon interaction cross-country section derived form the LLNL evaluated photon dated library, Z=1-50" Rad. Res.,Vol.6, part TO, Rev 4, Laurence Livemore Library, (1989). 